\title{
Identification of the amino acids in the major histocompatibility complex class II region of scottish blackface sheep that are associated with resistance to nematode infection
}

\begin{abstract}
Lambs with the Major Histocompatibility Complex DRB $1 * 1101$ allele have been shown to produce fewer nematode eggs following natural and deliberate infection. These sheep also possess fewer adult Teladorsagia circumcincta than sheep with alternative alleles at the DRB1 locus. However, it is unclear if this allele is responsible for the reduced egg counts or merely acts as a marker for a linked gene. This study defined the MHC haplotypes in a population of naturally infected Scottish Blackface sheep by PCR amplification and sequencing, and examined the associations between MHC haplotypes and faecal egg counts by generalised linear mixed modelling. The DRB $1 * 1101$ allele occurred predominately on one haplotype and a comparison of haplotypes indicated that the causal mutation or mutations occurred in or around this locus. Additional comparisons with another resistant haplotype indicated that mutations in or around the DQB2*GU191460 allele were also responsible for resistance to nematode infections. Further analyses identified six amino acid substitutions in the antigen binding site of DRB $1 * 1101$ that were significantly associated with reductions in the numbers of adult $\mathrm{T}$. circumcincta.
\end{abstract}

Keyword: Major histocompatibility complex; Sheep; Ovine; Haplotype scottish blackface; Nematode; Teladorsagia circumcincta 